Artículo

\title{
Estudio del agroecosistema con calabaza pipián en Cantarranas, Paso de Ovejas, Veracruz
}

\author{
Mónica Del Ángel-Ocampo \\ Arturo Pérez Vázquez ${ }^{\S}$ \\ Ciro Sánchez Hernández \\ Paola Carolina Taborda Bustillo \\ Eliseo García Pérez
}

${ }^{1}$ Colegio de Postgraduados-Campus Veracruz. Carretera Federal Veracruz-Xalapa km 88.5, Tepetates, Manlio Fabio Altamirano, México. CP. 91690. (angel.monica@colpos.mx; sanchez.ciro@colpos.mx; taborda.paola@colpos.mx; geliseo@colpos.mx).

${ }^{\S}$ Autor para correspondencia: parturo@colpos.mx.

\section{Resumen}

El objetivo fue analizar el agroecosistema de calabaza pipián para identificar problemas, eficiencia energética y financiera comparando producción de semilla seca a cuando se le da valor agregado (jamoncillo) en la comunidad de Cantarranas Municipio de Paso de Ovejas, Veracruz, México. Esta investigación se realizó en 2018, usando técnicas participativas y entrevistas semiestructuradas, análisis financiero y de productividad energética. El grado de interacción y comunicación entre agricultores fue analizado con el programa UCINET. Se encontró que el estado de Veracruz se ubica en séptimo lugar en la producción de semilla de pipián a nivel nacional, el municipio de Paso de Ovejas ocupa el decimoquinto lugar en el estado. El análisis de energía mostró una mayor productividad energética para el producto de valor agregado de pipián (jamoncillo) en contraste con la producción de producción de semilla. El análisis financiero mostró una baja rentabilidad para la producción de semilla (precio de 2017: Mex $\$ 20.00 \mathrm{~kg}^{-1}$ ). Se encontró una baja conectividad en la red de agricultores, lo que resulta en una comercialización individual generando bajos ingresos al productor. Es necesario adoptar formas más sostenibles de producción de calabaza pipián y promover una pequeña industria en la región.

Palabras clave: controlador cibernético, investigación participativa, potencial productivo.

Recibido: febrero de 2021

Aceptado: mayo de 2021 


\section{Introducción}

Los restos arqueológicos sugieren que la domesticación de Cucurbita argyrosperma Huber, comúnmente conocido como pipián o calabaza pipián, ocurrió en el centro de México. Los restos encontrados en el Valle de Tehuacán, Puebla, datan de hace más de 7000 años (Lira, 1995). La familia Cucurbitaceae en todo el mundo incluye 118 géneros y 825 especies. Según estudios taxonómicos y florísticos recientes, comprende 141 taxones: 128 silvestres y 13 cultivados (Lira et al., 2002).

Varias especies son importantes para la economía del agricultor como cultivo comercial y algunas otras tienen valor cultural entre varias comunidades rurales en México, debido a su uso como alimento y medicinal. Una de estas especies es Cucurbita argyrosperma (Lira et al., 2002), conocida comúnmente como calabaza pipián. La pulpa y cáscara de esta calabaza (subproducto) tienen un rendimiento entre 3.8-28 t en fresco por hectárea (Garza et al., 2010), que podrían ser útil en la alimentación pecuaria, ensilado o deshidratado. Asimismo, puede ser utilizado como fuente de pigmentos en dietas para aves ponedoras por su elevado contenido de carotenos (Dorantes-Jiménez et al., 2016).

Es importante destacar que la calabaza es un fruto de gran valor nutritivo por su elevado contenido de carbohidratos, fibra soluble, proteína, aceite de compuestos carotenoides, precursores de la vitamina $\mathrm{A}, \beta$ caroteno, criptoxantina y luteína (Hernández, 2009). Los frutos varían en tamaño, forma y color. El intervalo en el tamaño va de 14 a $50 \mathrm{~cm}$ de largo y de 14 a $25 \mathrm{~cm}$ de diámetro, piriforme o claviforme en la parte más delgada, tiene semillas de 1.5 a $3 \mathrm{~cm}$ de largo y 0.7 a 1.7 $\mathrm{cm}$ de ancho, planas, elípticas a lanceoladas (Dorantes-Jiménez et al., 2016). Normalmente, los productores prefieren para sembrar semillas con margen gris, delgado y uniforme, testa blanca ya que tienen mayor peso que las semillas de bordes anchos (Merrick y Bates, 1989).

Las semillas contienen $28 \%$ de aceite y $30 \%$ de proteína (FAO, 2018) y su consumo es frecuente en México y Centroamérica. Aparte de México, su cultivo se ha registrado en países como Guatemala, Honduras, El Salvador, Nicaragua, Costa Rica y Panamá (Hernández y León, 1994). El aceite de la semilla de calabaza proporciona grandes beneficios a la salud, siendo un aceite insaturado con un contenido de 60 al 90\% de ácido oleico y linoleico (Rossel et al., 2018).

Según estadísticas agrícolas del SIAP (2018), 16 estados en México se dedican a la producción de calabaza pipiana. El estado de Veracruz ocupa el séptimo lugar y el estado de Campeche el primero, con un total de 28665 ha cultivadas. En 2018, el área cultivada en Veracruz fue de 716 ha (SIAP, 2018). En el estado de Veracruz, 26 municipios cultivan calabaza pipián. Entre ellos, Paso de Ovejas, ubicado en la zona central del estado, ocupa el quinceavo lugar, con una superficie aproximada de 24 ha de temporal. Este cultivo es importante debido a la creciente demanda de semillas en el mercado local, estatal y nacional.

El cultivo de calabaza pipiana se cultiva preferentemente bajo condiciones de temporal en suelos arenosos y arcillosos con buen drenaje y contenido medio de materia orgánica, en climas de trópico húmedo y subhúmedo. En general, se siembra a finales del mes de mayo y principios de junio con la presencia de las primeras lluvias, y se cosecha de septiembre a diciembre (Garza et al., 2010). 
El concepto de agroecosistema se empieza a utilizar a mediados del siglo XX. Al respecto, Hart (1985), define al agroecosistema como un arreglo de componentes físicos, un conjunto o colección de cosas, unidas o relacionadas de tal manera que forman y actúan como una unidad, una entidad o un todo. Este concepto ha evolucionado y se entiende que el agroecosistema es un sistema que debe ser estudiado como una totalidad organizada (Martínez-Dávila y Casanova-Pérez, 2018), donde su entendimiento pasa por conocer su estructura y funcionamiento, y sus atributos sociales, económicos y ambientales en una apropiada escala y contexto (Pérez-Vázquez y Trinidad-Leyva, 2019). Es decir, que todo sistema tiene una estructura relacionada con el arreglo de sus componentes y tiene una función relacionada del cómo actúa el sistema.

Una investigación exploratoria de la región de interés permitió determinar los problemas prioritarios en cuanto a los cultivos de mayor importancia socioeconómica, resaltando el cultivo de calabaza pipián. Aunado a la escasa información científica de este cultivo bajo un enfoque de agroecosistemas. Por tanto, se planteó la necesidad de generar información científica sobre la importancia de este cultivo para la zona centro del estado de Veracruz que permitiera tener un entendimiento de su estructura y funcionamiento a diferentes niveles jerárquicos. El objetivo fue estudiar la situación actual del agroecosistema con calabaza pipiana en Cantarranas, Municipio de Paso de Ovejas, Veracruz.

\section{Materiales y métodos}

El enfoque de esta investigación fue un análisis mixto, cuantitativo y cualitativo del agroecosistema, realizado de febrero a abril de 2018. A nivel de Municipio, el estudio se realizó en Paso de Ovejas, Veracruz. A nivel de finca, se llevó a cabo en la comunidad de Cantarranas. La comunidad está ubicada a $220 \mathrm{~m}$ de altitud con un clima cálido subhúmedo (Aw0). La temperatura media anual es de $25.7^{\circ} \mathrm{C}$ y la precipitación media anual es de $1196 \mathrm{~mm}$. La selección de esta comunidad fue por la relevancia que tiene el cultivo de la calabaza pipián.

La información fue recolectada a través de la base de datos oficial SIAP (2018), para conocer el área cultivada, en tres niveles jerárquicos (cuenca, municipio y cultivo). Se visitó la comunidad de Cantarranas, tomando una muestra representativa de $20 \%$, que corresponde a 42 agricultores de pipián. Se analizó el flujo de entradas y salidas del agroecosistema mediante un análisis financiero y balance energético (Fluck, 1995). Se compararon dos sistemas de producción del cultivo de pipian, uno donde la producción de semilla que se comercializa seca y otra donde a la semilla se le da valor agregado (jamoncillo). Asimismo, se estudió el nivel de organización del controlador del agroecosistema en el territorio de interés (Figura 1).

Para diseñar el estudio se realizó un recorrido exploratorio para determinar los problemas prioritarios y considerar la actividad agrícola de mayor importancia y que aporta más ingresos a los agricultores. La comunidad de Cantarranas está ubicada a una altitud de $220 \mathrm{~m}$. El clima de la zona se define como subhúmedo tropical (Aw0) con una temperatura media anual de $25.7^{\circ} \mathrm{C}$ y una precipitación media anual de $1196 \mathrm{~mm}$. 


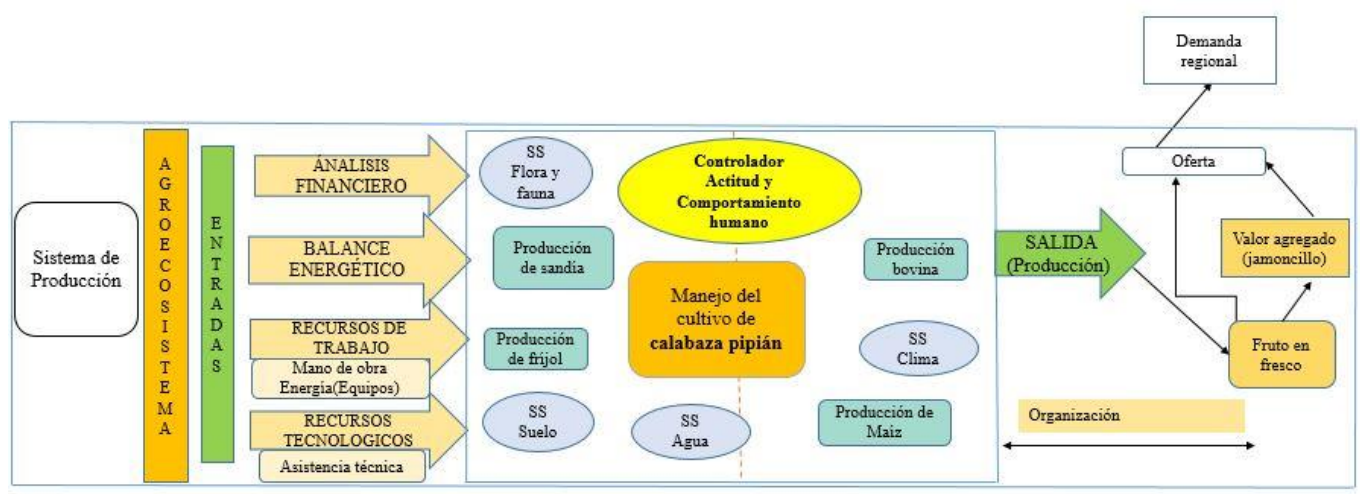

Figura 1. Modelo de agroecosistema con calabaza pipián en la región de Cantarranas, Paso de Ovejas Veracruz.

A nivel de cuenca, se utilizó el programa QGIS para identificar las áreas de mayor aptitud agroecológica para cultivar pipián. A nivel municipal, se realizaron entrevistas semiestructuradas con actores clave (Representante Municipal de Desarrollo Agropecuario y Jefe Distrital 006-La Antigua) y obtener información sobre la producción de pipián. A nivel de cultivo, se utilizaron técnicas de investigación participativa (entrevista semiestructurada, lluvia de ideas, árbol de problemas, clasificación) y un cuestionario. En reunión con 42 productores de calabaza pipián de la comunidad de Cantarranas, Veracruz y usando diversas dinámicas participativas se identificaron y priorizaron problemas, en presencia de la autoridad ejidal.

Para obtener la red de colaboración y el nivel de comunicación entre los agricultores, se utilizó el software UCINET. Se determinaron tres medidas de centralidad: rango, intermediación y grado de cercanía; utilizando una red gráfica, se establecieron varias relaciones, incluido el porcentaje de densidad (Kuz, 2016). A través de una entrevista se recabo información en la comunidad de La Ternera, Veracruz, para describir el valor agregado (dulce de jamoncillo). Se utilizó un cuestionario estructurado para el análisis financiero, recolectando datos de costos e ingresos por producción de semilla y valor agregado (jamoncillo). Los indicadores financieros estimados fueron: relación beneficio/costo (B/C), valor actual neto (VPN) y tasa interna de rendimiento (TIR) (Infante, 1998).

El mismo cuestionario se usó para hacer los presupuestos de energía, registrando entradas y salidas (producción) del sistema agrícola en términos de energía directa e indirecta. Se asignaron valores de energía en unidad térmica británica (BTU) a todas las entradas y salidas. Para el caso de la producción de jamoncillo, se registraron los costos e insumos utilizados para su producción. La productividad energética se estimó según la metodología de Fluck (1995); Pardo (1987).

\section{Resultados y discusión}

\section{Calabaza pipián a nivel estatal y municipal}

Según SIAP (2018), los estados con mayor potencial para la producción de calabaza pipiana son: Veracruz, Campeche, Yucatán, Tamaulipas, parte de Oaxaca, Michoacán, Jalisco, Nayarit y Sinaloa (Figura 2). La calabaza pipiana prospera en regiones tropicales y subtropicales, se distribuye en una amplia gama de altitudes, desde el nivel del mar hasta los $1800 \mathrm{~m}$ (Ayvar et al. (2007). A nivel estatal, su cultivo se concentra en el norte y centro del estado y parcialmente el sur. 


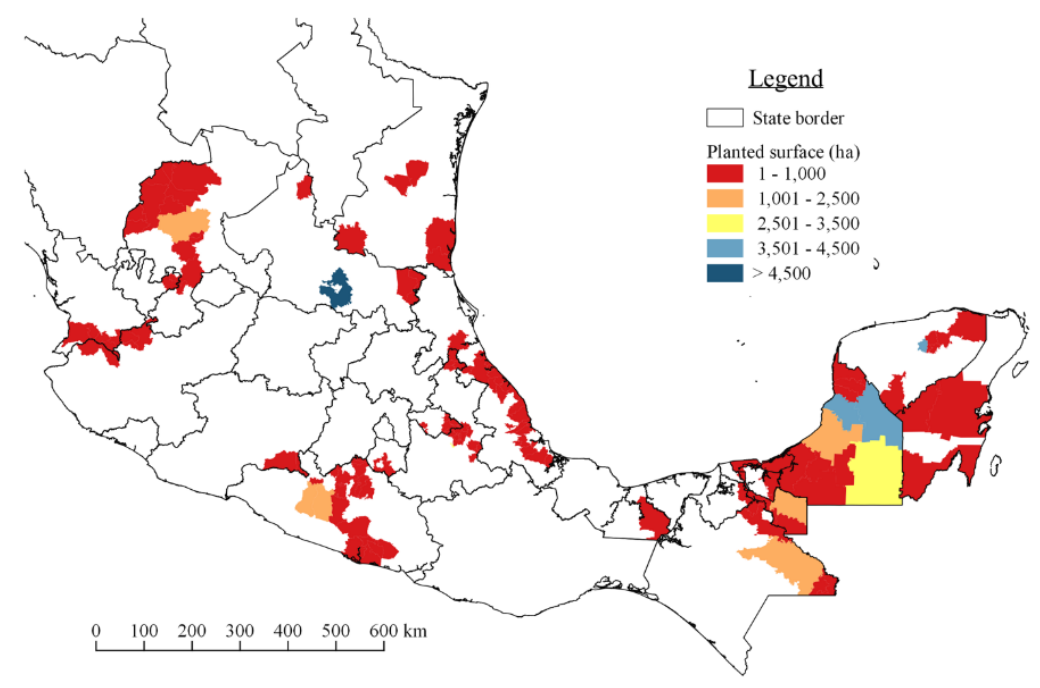

Figura 2. Estados con mayor potencial productivo para el cultivo de la calabaza pipián $(C$. argyrosperma).

El municipio de Paso de Ovejas se encuentra en el decimoquinto lugar en producción con un área cultivada de 24 ha. La producción también se concentra en la parte central, particularmente en los municipios de Alto Lucero, Actopan, La Antigua, Puente Nacional, Paso de Ovejas y Úrsulo Galván (SIAP, 2018). Las comunidades más importantes en el municipio de Paso de Ovejas que cultivan calabaza pipián son: Mata Mateo, Cantarranas, Acazónica, Angostillo, así como La Ternera y Mata de Jobo que pertenecen al municipio Puente Nacional (Figura 3).

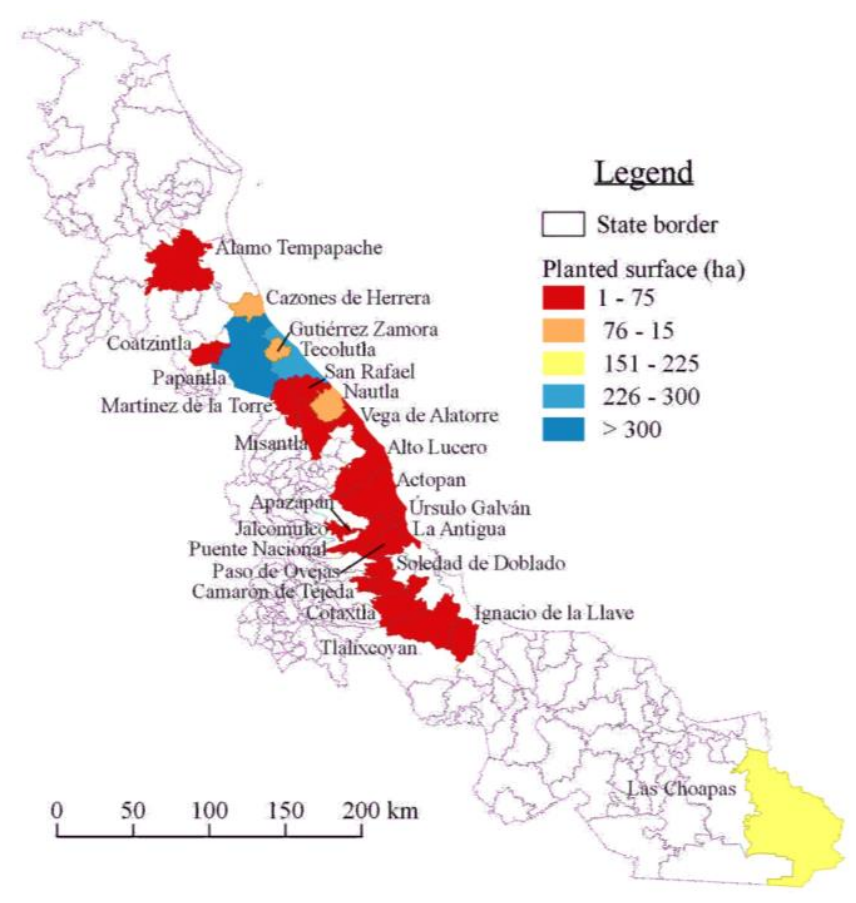

Figura 3. Municipalidades del estado de Veracruz con el mayor potencial productivo para el cultico de la calabaza pipián (C. argyrosperma). 
Los agricultores señalaron: 'hace aproximadamente 15 años, solía cultivar calabaza pipián asociada con maíz y papaya, pero la calabaza pipián ha generado más ingresos por lo que ahora la sembramos como monocultivo, para obtener mayor producción'. Sin embargo, su monocultivo ha generado mayor incidencia de plagas y enfermedades.

En diversos lugares de México, el cultivo de calabaza pipián es importante en los sistemas agrícolas tradicionales (Whitaker y Davis, 1962). El sistema de producción tradicional involucra la asociación principalmente de maíz (Zea mays), calabaza (Cucurbita argyrosperma) y frijol (Phaseolus vulgaris). También representa un bagaje de conocimiento tradicional y prácticas de conservación de la diversidad genética de cultivos adaptados a su medio ambiente, suelos y factores climáticos, y preferencia cultural y socioeconómica regional (Canul et al., 2005).

Tal como lo comenta Ebel et al. (2017) la asociación de cultivos de maíz, frijol y calabaza, lo que subraya el potencial productivo de los sistemas de producción diversificado. Además del cultivo de calabaza pipián, durante la temporada de lluvias, algunos agricultores cultivan sandía, maíz y papaya. Sin embargo, se carece de un paquete tecnológico que contribuya a elevar la productividad y la rentabilidad del cultivo. Además, no hay suficiente información respecto a los factores que limitan la cadena productiva de la semilla de pipián en la elaboración de platillos y dulces.

Porta et al. (2003), comentan que uno de los factores que limitan los bajos rendimientos en la producción son los relacionados con las características fisicoquímicas del suelo. Al respecto, la fertilización foliar es un método eficiente como complemento de la fertilización al suelo, especialmente en las etapas de crecimiento que se ve reflejado en el rendimiento del cultivo sobre todo en la producción de semillas secas (Díaz-Nájera et al., 2015). Dado que el ciclo de cultivo de la calabaza pipiana dura únicamente tres meses, desde su siembra hasta la cosecha, su monocultivo puede ocasionar la disminución de nutrientes en el suelo (Ayvar et al., 2007).

\section{Problemática regional del cultivo de calabaza pipián}

Los resultados del árbol de problemas, arrojó que la principal amenaza de este cultivo es la sequía, debido a la eventualidad de las lluvias, cada vez más escasas y mal distribuidas durante la temporada. Otro de los problemas es la variación en el precio de la semilla (una vez cosechada). En 2014, el precio fue de $\$ 41.00 \mathrm{~kg}^{-1}$ (pesos mexicanos) y fue una cosecha bastante rentable, pero a partir de 2017 el precio ha sido de $\$ 18.00-20.00 \mathrm{~kg}^{-1}$, precio que solo cubre los costos mínimos de producción.

Los agricultores comentan: 'este precio se debe a que actualmente la producción total es acaparada por un grupo organizado, quien tiene contrato de compra-venta con otro proveedor'. Otro problema identificado fue una reducción de los rendimientos al estar continuamente sembrando en las mismas parcelas bajo el sistema de monocultivo. Esto genera a los productores altos costos de producción, debido a la fertilización convencional y control de plagas y enfermedades. Un problema son las plagas y enfermedades, una larva (Spodoptera exigua Hübner) que perfora el fruto tierno y el virus del mosaico que afecta la capacidad fotosintética del cultivo y el rendimiento.

Los frutos se cosechan en agosto y las semillas se secan a lo largo de la carretera durante un par de días soleados. En caso de tener una lluvia imprevista durante el secado de la semilla, esto ocasiona que se humedezcan, provocando pérdida en la calidad y apariencia de la semilla. Se reporta que el 
uso de Bayfolan Forte incrementa el rendimiento en calabaza pipián hasta $50 \%$, debido a su composición en macro y micronutrientes, lo cual podría ser una alternativa para reducir costos de fertilización (Díaz-Nájera et al., 2015).

Sequi (2004), menciona que la aplicación de micronutrientes vía foliar se ha convertido en una práctica importante para los productores, debido a que corrige las deficiencias nutrimentales de las plantas, favorece el buen desarrollo de los cultivos, además de mejorar el rendimiento y la calidad comercial. Es importante mencionar que no se cuenta con técnicos que asesoren a los productores y los capaciten, en la producción de calabaza pipián, principalmente en la implementación de prácticas agroecológicas más amigables con el ambiente, así como en temas de agregación de valor.

Sin duda esto mejoraría considerablemente los sistemas de producción agrícola e ingresos del productor. Por ejemplo, los productores no realizan podas a su cultivo, pues si se elimina la dominancia apical en los tallos rastreros primarios y secundarios, podría promover el desarrollo de guías laterales cortas y precoces, con mayor fructificación, y les permitiría mayores densidades de siembra e incrementar el rendimiento y la rentabilidad del cultivo (Ayvar et al., 2004).

El cultivo de calabaza pipián se da preferentemente en suelos franco arenoso y franco arcilloso con buen drenaje y contenido medio de materia orgánica. Normalmente, los productores usan semillas de borde fino porque tienen más peso que las semillas de borde ancho. El cultivo presenta plagas importantes, esto debido a que dichas plagas han creado resistencia a los agroquímicos que se aplican. El total de productores afirmaron tener problemas con el gusano verde (Diaphania hyalinata L. o D. nitidalis Stoll) siendo barrenadores de las guías y de los frutos tiernos; que invernan como pupa en el suelo (Bautista y Vejar, 1999).

Por tanto, para controlar las plagas y enfermedades, los agricultores deben usar una amplia gama de agroquímicos con valores de hasta $\$ 750.00$ por ciclo de cultivo. El control de malezas se basa en la aplicación de herbicidas, preferiblemente dos veces por ciclo de cultivo. La primera después de la siembra y la segunda cuando la guía es aún manejable (1 m de longitud).

Tan pronto aparecen los frutos de la calabaza, aplican agroquímicos como Denim 19 CE o Furadan 350 L. El problema del mildiu polvoriento (Erysiphe cichoracearum DC) es más grave durante condiciones de sequía. El área promedio cultivada por agricultor es de 1-10 ha. El rendimiento promedio reportado es de alrededor de $600-700 \mathrm{~kg} \mathrm{ha}^{-1}$. Esto coincide con, George (1999), quien señala que el rendimiento promedio oscila entre $0.5 \mathrm{y} 1 \mathrm{t} \mathrm{ha}^{-1}$.

\section{Redes sociales de la comunidad de Cantarranas, Paso de Ovejas Veracruz}

La edad promedio de los agricultores fue de 62 años. En el análisis de la red, la densidad del factor social fue $1.6 \%$ de la interrelación entre agricultores de Cantarranas, lo que corresponde a un bajo nivel de interacción. Se determinaron trece subgrupos, lo que significa que la mayoría de los productores trabajan solos con una mínima intercomunicación. Dos agricultores (Adán S y Pedro H) tuvieron el mayor grado de intermediación y referente.

Otros dos agricultores (Mario Q y Antonio D) tuvieron el mayor grado de cercanía y por tanto, tienen la mayor capacidad para acceder a los demás nodos de la red (Figura 4). Mario Q obtuvo el mayor grado de cercanía e intermediación dentro de la comunidad y es uno de los actores por apoyar en servicios de capacitación, transferencia de tecnología, comunicación de información. 


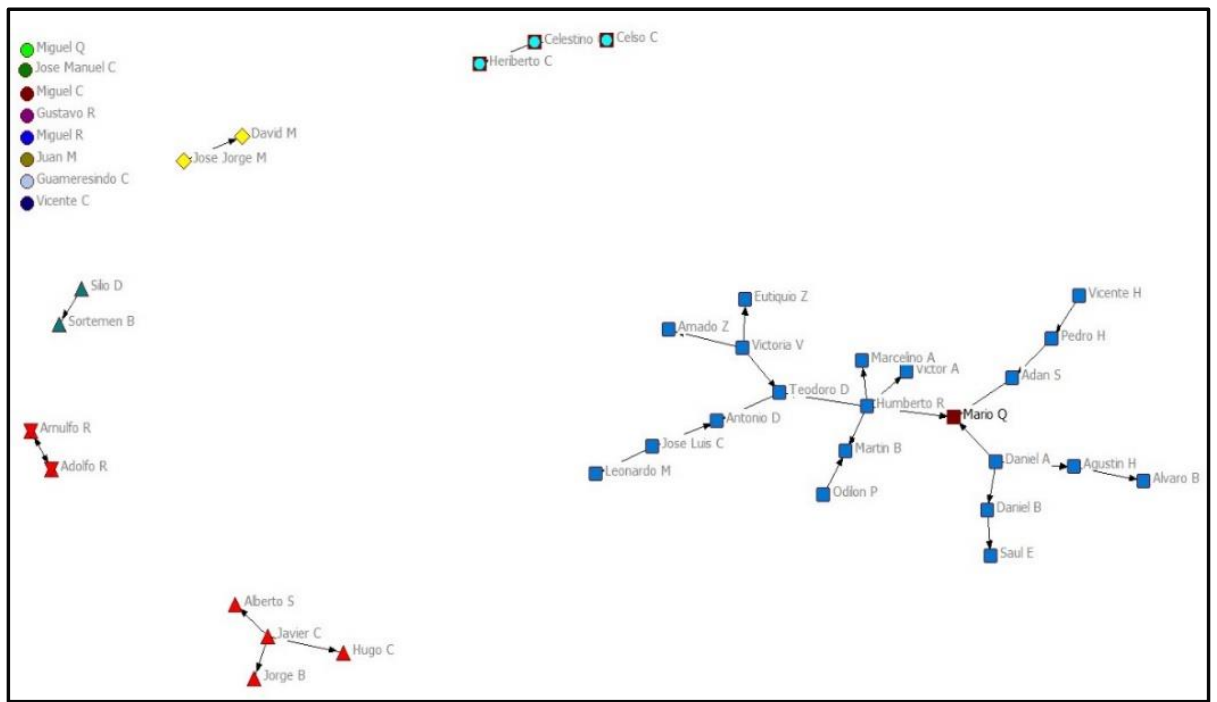

Figura 4. Análisis de la red de agricultores de la comunidad de Cantarranas, Paso de Ovejas, Veracruz.

Los actores de la red adquieren roles sociales de acuerdo con la forma y tipo de insumos que manejan, por lo que llegan a cumplir funciones específicas, en aspectos organizacionales, gerentes, innovadores, diseñadores, adoptadores, difusores, integradores, confianza, entre otros (Nuñez, 2008). Si los productores del agroecosistema calabaza pipián no tienen una organización adecuada no pueden insertarse en el mercado (Ireta et al., 2018).

\section{Costos de producción e indicadores financieros del agroecosistema calabaza pipián}

Actividades como secar la semilla, limpiar la tierra, barbecho, chapeo, fertilizar y aspergar agroquímicos tienen el mayor peso en el costo total de producción (Figura 5). Esto coincide con Ireta et al. (2018), quienes señalaron que preparación del suelo, semilla, fertilización, herbicida, control fitosanitario, mano de obra; tienen el mayor impacto en el costo total de producción.

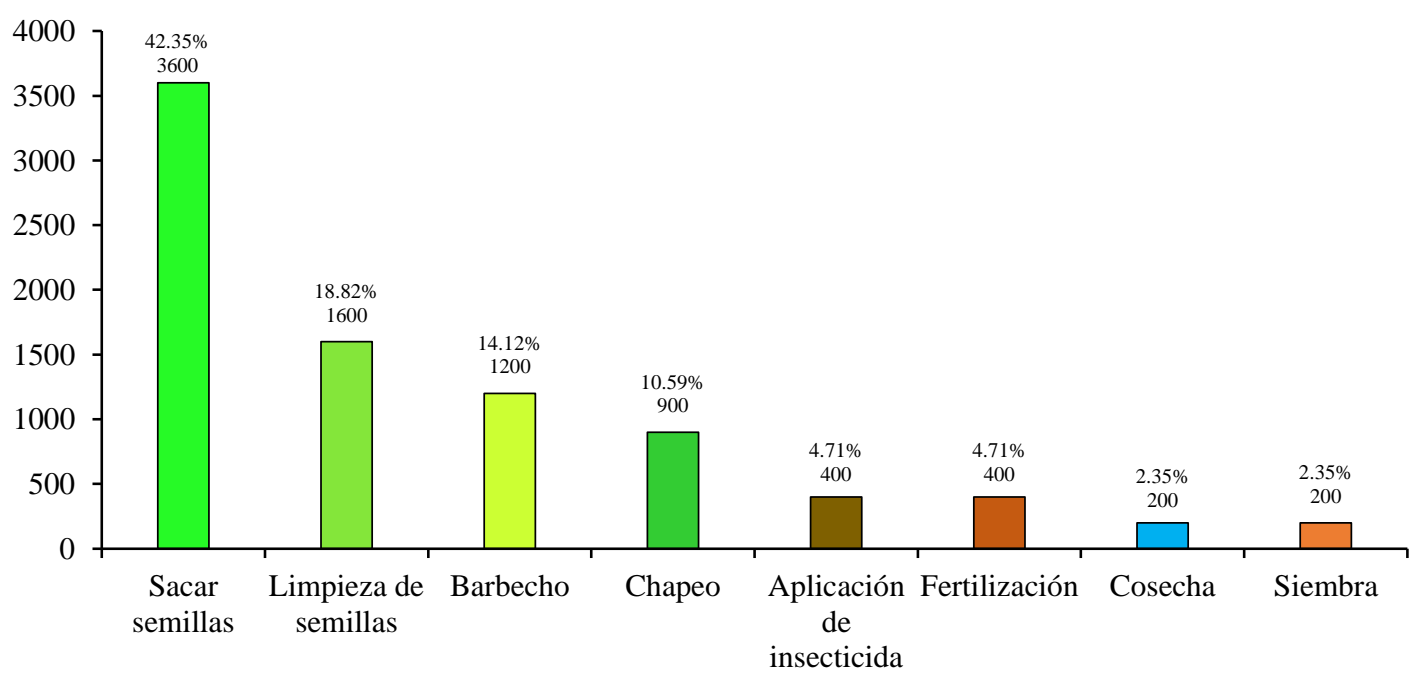

Figura 5. Distribución de los costos de producción en el cultivo de calabaza pipián. 
Los indicadores financieros (punto de equilibrio, relación costo/beneficio) de la semilla de pipián con valor agregado fueron más altos en comparación con el valor de la semilla de pipián seco. Los resultados indican que el punto de equilibrio como semilla seca, es Mex $\$ 22.00 \mathrm{~kg}^{-1}$. Sin embargo, cuando se agrega valor a la semilla y se venden como dulce de jamoncillo, se obtiene un ingreso más alto (Cuadro 1), con una relación costo/beneficio de 1.03.

Cuadro 1. Indicadores financieros de producción en fresco y valor agregado en calabaza pipián por ciclo anual.

\begin{tabular}{ccccc}
\hline Tipo de producción & Costo $\left(\$ \mathrm{ha}^{-1}\right)$ & B/C & VAN & TIR \\
\hline Semilla de pipián (seco) & 10570.00 & 0.13 & 1430.00 & $8 \%$ \\
Jamoncillo (dulce) & 47370.00 & 1.03 & 20276.00 & $22 \%$ \\
\hline
\end{tabular}

\section{Balance energético de producción de semilla seca y como valor agregado (jamoncillo)}

La preparación de procesos agroindustriales como el jamoncillo se lleva a cabo de manera tradicional por familias de Mata de Jobo y es su principal fuente de ingresos. Un informante clave dijo que la venta se lleva a cabo durante todo el año, pero la mayor demanda se da en septiembre y octubre, con las festividades de 'Día de muertos'.

La energía total utilizada para semilla seca es menor que la del producto de valor agregado (Cuadro 2). El mercado regional de derivados del pipián (jamoncillo y salsa pipián) incluye mercados municipales, tiendas bien establecidas y postores ambulantes en las ciudades de Xalapa, Huatusco, Coscomatepec, Veracruz y Cardel, que incluye un precio de transporte de 100-150 pesos.

Cuadro 2. Resultado del balance energético de la calabaza pipiana como producto fresco y con valor agregado (jamoncillo).

\begin{tabular}{|c|c|c|c|c|c|c|}
\hline \multirow{2}{*}{ Entradas } & \multirow{2}{*}{ Unidades } & \multirow{2}{*}{ Cantidad- } & \multicolumn{3}{|c|}{ Energía total } & \multirow{2}{*}{$\begin{array}{c}\text { Productividad } \\
\text { energética }\end{array}$} \\
\hline & & & Ambos productos & Semilla & Jamoncillo & \\
\hline Tractor & Btu $(\$)$ & 2100 & 27747300 & & & \\
\hline Nitrógeno & Btu (kg) & 1 & 72156 & & & \\
\hline Herbicida & Btu (kg) & 4 & 1079162 & & & \\
\hline Insecticida & Btu (kg) & 2 & 438628 & & & \\
\hline Jornales & Btu (h) & 328 & 17755624 & & & \\
\hline Subtotal & & & & 47092870 & & 6.795E-06 \\
\hline Leña & Btu (kg) & 320 & 5713920 & & & \\
\hline Otros costos & Btu Mex (\$) & 0.03 & 400221770 & & & \\
\hline Subtotal & & & & & 405935690 & $7.883 \mathrm{E}-07$ \\
\hline Total & Btu & & 453028561 & & & $7.06 \mathrm{E}-07$ \\
\hline
\end{tabular}




\section{Conclusiones}

El cultivo de la calabaza pipiana en el área central del estado de Veracruz (municipios de Paso de Ovejas y Puente Nacional) tiene una gran importancia en la economía de los productores. Sin embargo, en los últimos años, presenta problemas importantes que ponen en riesgo su permanencia como monocultivo, esto debido a la caída del precio, eventualidad y escasez de lluvias, plagas y enfermedades y baja organización de los productores para insertarse en el mercado de esta semilla. La agregación de valor puede ser una opción para lograr mayores ingresos.

El abandono del cultivo tradicional del pipián (en asocio con maíz y papaya) puede ser una opción que requiere ser valorada desde las diversas dimensiones, social, financiera y ambiental. Sin embargo, la práctica del monocultivo está representando un alto costo de producción, reduciendo las ganancias. En lo que se refiere al factor social, se encuentra que la densidad fue de $1.6 \%$ de la interrelación entre los productores, lo que corresponde a un bajo nivel de conectividad y por tanto bajo nivel de organización.

El análisis financiero mostró una baja rentabilidad para la semilla seca (precio en 2017 de Mex $\$ 20.00 \mathrm{~kg}^{-1}$ ) en comparación con valor agregado (dulce de jamoncillo). El esquema de valor agregado tuvo una mayor productividad energética, respecto a la producción de la semilla de pipián seca. Se recomienda que se deben explorar alternativa del uso del subproducto de la calabaza pipián (la pulpa y cáscara de calabaza) en la alimentación animal, principalmente en el ganado bovino, porcino y aves.

\section{Agradecimientos}

Al Consejo Nacional CONACYT, por la beca otorgada a la primera autora. Al Colegio de Postgraduados, Campus Veracruz, por el apoyo financiero brindado. A los productores y autoridades de las comunidades de Cantarranas y La Ternera por su colaboración e información.

\section{Literatura citada}

Ayvar, S. S.; Bahena, M. A.; Doribel, C. M.; Durán, R. J. A. y De Luna, M. J. G. 2004. Rendimiento de la calabaza pipiana en respuesta a la poda y la densidad de población. Rev. Fitot. Mex. 27(1):69-72.

Ayvar, S. S.; Mena, A. V.; Durán, R. J. A.; Cruzaley, R. S. y Gómez, M. N. O. 2007. La calabaza pipiana y su manejo integrado. Instituto Nacional de Investigaciones Forestales, Agrícolas y Pecuarias (INIFAP)- CBTa 176, Fundación Produce de Guerrero AC, Campo Experimental Iguala y CSAEGRO. Iguala, Guerrero, México. 26 p.

Bautista, M. N. y Vejar, G. C. 1999. Lepidópteros más comunes en las hortalizas. In: hortalizas, plagas y enfermedades. Anaya, R. S. y Romero, J. N. (Ed.). Editorial Trillas. México, DF. 255-281. p

Canul, K. J.; Ramírez, P. V.; Castillo, F. G. y Chávez, S. J. L. 2005. Diversidad morfológica de calabaza cultivada en el centro-oriente de Yucatán, México. Rev. Fitot. Mex. 28(4):339-349.

Díaz-Nájera, J. F.; Michel-Aceves, A. C.; Ayvar-Serna, S.; Alvarado-Gómez, O. G.; DuránRamírez, J. A.; Tejeda-Reyes, M. A.; Solís-Aguilar, J. F. y Díaz-Ceniceros, H. L. 2015. Fertilización foliar en calabaza pipiana (Cucurbita argyrosperma Huber) en Apipilulco, Guerrero. Agraria. 12(3):101-110. 
Dorantes-Jiménez, J.; Flota-Bañuelos, C.; Candelaria-Martínez, B.; Ramírez-Mella, M. y CrosbyGalván, M. M. 2016. Calabaza Chihua (Cucurbita argyrosperma Huber), alternativa para alimentación animal en el trópico. Agro Productividad. 9(9):33-37. file:///c:/users/usuario/ downloads/agroproductividadixoctubre2016baja.pdf.

Ebel, R.; Pozas, C. J. G.; Soria, M. F. y Cruz, G. J. 2017. Manejo orgánico de la milpa: rendimiento de maíz, frijol y calabaza en monocultivo y policultivo. Terra Latinoam. 35(2):149-160.

FAO. 2018. Organización de las naciones unidas para la alimentación y la agricultura. http://www.fao.org/tempref/GI/Reserved/FTP_FaoRlc/old/prior/segalim/prodalim/ prodveg/cdrom/contenido/libro11/cap2.htm.

Fluck, R. C. 1992. Energy in Farm Production, Energy in World Agriculture, 6, Amsterdam. Elsevier. 13-52 pp.

Garza, O. S.; Núñez, G. H. C.; Serrano, E. A.; Huez, L. M. A. y López, E. J. 2010. Comportamiento diferenciado de líneas, híbridos y criollos de calabaza arota (Cucurbita argyrosperma Huber) en primavera y otoño. Biotecnia. 12(3):3-13.

George, R. A. T. 1999. Vegetable seed production. $2^{\text {nd }}$ (Ed.). Cabi Publishing. USA. 328 p.

Hart, R. D. 1985. Conceptos básicos sobre agroecosistemas. Catie. Turrialba, CR. 161 p.

Hernández, B. J. E. and León, J. 1994. Neglected crops 1492 from a different perspective. Food and agriculture organization of the United Nations. FAO plant production and protection series No. 26. Published in collaboration whit the Botanical Garden of Córdoba, Spain, as part of the etnobotánica 92 programme (Andalusia, 1992). $348 \mathrm{p}$.

Hernández, M. S. 2009. Multiplicación in vitro vía organogénesis en calabaza. Agronomía Mesoam. 20(1):11-22.

Infante, V. A. 1998. Evaluación financiera de proyectos de inversión. (Ed.). Norma. Bogotá, Ciudad de México, 5. Panamá, San Juan Barcelona Caracas. 401 p.

Ireta, A. R.; Pérez-Hernández, P.; Bautista-Ortega, J. y Rosas-Herrera, E. L. 2018. Análisis de la red de calabaza chihua (Cucurbita argyrosperma Huber) en Campeche, México. Agrociencia. 52(1):151-167. https://www.researchgate.net/publication/323129044.

Martínez-Dávila, J. P. and Casanova-Pérez, L. 2018. Epistemic and conceptual orphanhood in the sustainability of agroecosystems. Sustainability of Agroecosystems. 1(1):1-16.

Merrick, L. C. and Bates, D. M. 1989. Classification and nomenclature of Cucurbita argyrosperma. Baileya. 23(2):94-102.

Kuz, A.; Falcol, M. y Giandini, R. 2016. Análisis de redes sociales: un caso práctico. Computación y sistemas. 20(1):89-106. Doi: 10.13053/CyS-20-1-2321.

Lira, S. R.; Villaseñor, J. L. and Ortiz, E. 2002. A proposal for the conservation of the family cucurbitaceae in México. Biodivers. Conserv. 11(10):1699-1720. Doi: 10.1023/A: 1020303905416.

Lira, S. R. 1995. Estudios taxonómicos y eco geográficos de las Cucurbitaceae Latinoamericanas de importancia económica. Systematic and ecogeographic studies on crop genepools. 9. International Plant Genetic Resources Institute. Roma, Italia. http://www.fao.org/library/ library-home/en/.

Núñez, E. J. F. 2008. Exploración en la operación y modelización de redes sociales de comunicación para el desarrollo rural en zonas marginadas de Latinoamérica. Estudios de casos: red nacional de desarrollo rural sustentable (Rendrus) y red iniciativa de nutrición humana. Tesis doctoral. Universidad Politécnica de Cataluña, Cátedra UNESCO en sostenibilidad. Barcelona, España. 7-15 pp. 
Pérez-Vázquez, A. y Leyva-Trinidad D. A. 2019. Análisis comparativo de los principales enfoques de investigación de los sistemas agrícolas. Agro Productividad. 12(6):31-37.

Porta, C. J.; López-Acevedo, M. R. y Roquero, C. D. 2003. Edafología para la agricultura y el medio ambiente. Editorial Mundi Prensa. $3^{\mathrm{a}}$ (Ed.). Madrid, España. 928 p.

Pardo, R. 1987. Small-scale forest enterprises. AO Food and agriculture organization of the united nations, Rome. Inter. J. Forestry Forest Industries. 39(3):157-158. http://www.fao.org/ docrep/s4550e/s4550e00.htm.

Rossel, K. D.; Ortiz, H. L.; Amante, A. O.; Durán, G. H. M. y López, M. L. A. 2018. Características físicas y químicas de la semilla de calabaza para mecanización y procedimiento. Rev. Investigación de la Universidad de la Salle Bajío. 10(2):61-77.

SIAP. 2018. Servicio de Información Agroalimentaria y Pesquera. Secretaría de Agricultura, Ganadería, Desarrollo Rural, Pesca y Alimentación. Cierre de producción agrícola por cultivo http://nube.siap.gob.mx/cierreagricola/.

Sequi, P. 2004. Los micro elementos en la nutrición vegetal. Instituto experimental para la nutrición de las plantas. $3^{\mathrm{a}}$ (Ed.). Ediciones Mundi Prensa. Italia. 78 p.

Whitaker, T. W. and Davis, G. N. 1962. Cucurbits, botany, cultivation and utilization. Interscience. Publishers, Inc. New York. 249 p. 